\title{
The autoregulator receptor homologue AvaR3 plays a regulatory role in antibiotic production, mycelial aggregation and colony development of Streptomyces avermitilis
}

Correspondence
Takuya Nihira
nihira@icb.osaka-u.ac.jp

Received 18 January 2011

Revised 9 May 2011

Accepted 21 May 2011

\author{
Kiyoko T. Miyamoto, ${ }^{1}$ Shigeru Kitani, ${ }^{1}$ Mamoru Komatsu, ${ }^{2}$ Haruo Ikeda ${ }^{2}$ \\ and Takuya Nihira ${ }^{1,3}$ \\ ${ }^{1}$ International Center for Biotechnology, Osaka University, 2-1, Yamadaoka, Suita, Osaka \\ 565-0871, Japan \\ ${ }^{2}$ Kitasato Institute for Life Sciences, Kitasato University, 1-15-1, Kitasato, Sagamihara, Kanagawa \\ 252-0373, Japan \\ ${ }^{3} \mathrm{MU}-\mathrm{OU}$ Collaborative Research Center for Bioscience and Biotechnology, Faculty of Science, \\ Mahidol University, Rama VI Rd, Bangkok 10400, Thailand
}

\begin{abstract}
The $\gamma$-butyrolactone autoregulator receptor has been shown to control secondary metabolism and/or morphological differentiation across many Streptomyces species. Streptomyces avermitilis produces an important anthelmintic agent (avermectin) and two further polyketide antibiotics, filipin and oligomycin. Genomic analysis of $S$. avermitilis revealed that this micro-organism has the clustered putative autoregulator receptor genes distant from the antibiotic biosynthetic gene clusters. Here, we describe the characterization of avaR3, one of the clustered receptor genes, which encodes a protein containing an extra stretch of amino acid residues that has not been found in the family of autoregulator receptors. Disruption of avaR3 resulted in markedly decreased production of avermectins, with delayed expression of avermectin biosynthetic genes, suggesting that AvaR3 positively controls the avermectin biosynthetic genes. Moreover, the disruption caused increased production of filipin without any changes in the transcriptional profile of the filipin biosynthetic genes, suggesting that filipin production is indirectly controlled by AvaR3. The avaR3 disruptant displayed fragmented growth in liquid culture and conditional morphological defects on solid medium. These findings demonstrated that AvaR3 acts as a global regulator that controls antibiotic production and cell morphology.
\end{abstract}

\section{INTRODUCTION}

The genus Streptomyces consists of Gram-positive, soildwelling bacteria characterized by a complex developmental life cycle and the ability to produce a variety of secondary metabolites, including antibiotics with important applications in human and veterinary medicine, and agriculture. The initiation of secondary metabolism typically coincides with the post-exponential phase in liquid-grown culture and with the onset of aerial mycelium formation on solid culture. One of the well-known regulatory systems for secondary metabolism is the signalling system with the $\gamma$-butyrolactone autoregulator as a small diffusible signalling molecule (Bibb, 2005; Takano, 2006). In this system, binding of the autoregulator

Abbreviations: HTH, helix-turn-helix; qRT-PCR, quantitative RT-PCR; RACE, rapid amplification of 5' cDNA ends.

Six supplementary figures and a supplementary table are available with the online version of this paper. to its cognate receptor causes the receptor to lose DNAbinding activity toward autoregulatory elements (AREs), which are frequently located in the promoter regions of target genes (Folcher et al., 2001), and in turn activates transcription of the target genes, which results in the coordinated expression of regulatory and biosynthetic genes involved in secondary metabolism. The autoregulator signalling system is involved not only in the onset of biosynthesis of secondary metabolites, but also occasionally in the onset of morphological differentiation (Folcher et al., 2001; Healy et al., 2009; Horinouchi, 2007).

To date, many genes encoding autoregulator receptors and their homologues have been reported to control antibiotic production, and the following three groups are recognised based on their gene loci and properties in the regulatory system. First, an autoregulator receptor gene, often accompanied by genes encoding a homologue of the autoregulator receptor, is found within an antibiotic 
biosynthetic gene cluster, and both of them operate at several regulation layers of biosynthesis of the cognate antibiotic. This type of regulation is regarded as a pathway-specific system for antibiotic production. In Streptomyces virginiae, BarA, a $\gamma$-butyrolactone autoregulator receptor, directly controls the transcription of $b a r B$, which encodes a receptor homologue (Kinoshita et al., 1997; Nakano et al., 2000). Multiple regulatory genes under the control of BarA and BarB coordinately regulate the expression of virginiamycin biosynthetic genes, which lie in the flanking regions of the barA and barB genes (Matsuno et al., 2004; Pulsawat et al., 2007, 2009). Second, a gene encoding an autoregulator receptor in the gene cluster for antibiotic biosynthesis simultaneously controls production of both the corresponding antibiotic and another antibiotic whose biosynthetic genes are distal to the locus of the receptor gene. Representative regulators of this type are ScbR (SCO6265) and ScbR2 (SCO6286) of Streptomyces coelicolor A3(2), which regulate the expression of a cryptic type I polyketide biosynthetic gene cluster (SCO6269-SCO6288) by repressing a pathway-specific regulatory gene, and indirectly stimulate production of actinorhodin and undecylprodigiosin (Gottelt et al., 2010; Takano et al., 2005). Third, an autoregulator receptor gene has no adjacent biosynthetic gene clusters but regulates the expression of antibiotic biosynthetic genes that are located apart from the locus of the receptor gene, as exemplified by ArpA of Streptomyces griseus. ArpA modulates self-resistance and biosynthesis of streptomycin and other metabolites in concert with aerial mycelium formation by controlling the pleiotropic transcriptional regulator AdpA in the A-factor regulatory cascade (Horinouchi, 2007).

Streptomyces avermitilis produces a family of polyketide macrocyclic lactones known as avermectins, which have potent antiparasitic and broad-spectrum activities against nematodes and arthropod parasites (Burg et al., 1979), together with two other families of polyketide antibiotics, filipins and oligomycins. The biosynthetic pathways of the three types of antibiotic have been predicted and partially elucidated (Ikeda et al., 1999; Omura et al., 2001), while genetic information on the regulatory mechanism of antibiotic production has so far been limited. With respect to avermectin production, we recently reported that AveR, which is encoded by a gene situated on the left-hand extremity of the avermectin biosynthetic gene cluster, is a positive LAL-family regulator for controlling avermectin biosynthetic genes (Kitani et al., 2009). Further understanding of the mechanisms by which avermectins and other antibiotics are regulated is necessary to achieve a high-yield production of antibiotics as well as to arrest the production of unwanted secondary metabolites. In this study, we characterized avaR3, which encodes a $\gamma$-butyrolactone autoregulator receptor homologue, and demonstrated that AvaR3 functions as a global regulator for avermectin production and cell morphology both in liquid culture and on solid medium.

\section{METHODS}

Bacterial strains, plasmids and growth conditions. S. avermitilis KA320 (isogenic to MA-4680) was obtained from the culture collection of the Kitasato Institute. Escherichia coli DH5 $\alpha$ was used for general DNA manipulation, and E. coli $\mathrm{F}^{-} d c m \Delta(\mathrm{srl}-$ recA)306:: Tn 10 carrying pUB307-aph:: Tn7 was used for E. colil Streptomyces conjugation. E. coli strains were grown in $2 \times$ yeast extract-tryptone (YT) medium, with appropriate antibiotics when necessary. pKU451, pKU474 (Supplementary Fig. S1) and pKU250 (Komatsu et al., 2010) were used to construct a vector for gene disruption. For complementation, pENTR (Invitrogen) was used for DNA cloning, and pSET152 (Bierman et al., 1992) was used to introduce DNA into Streptomyces. For DNA manipulation in Streptomyces, standard procedures were performed according to Kieser et al. (2000). The Streptomyces strains were grown at $28{ }^{\circ} \mathrm{C}$ on YMS medium (Ikeda et al., 1987) supplemented with $10 \mathrm{mM} \mathrm{MgCl}_{2}$ and $10 \mathrm{mM} \mathrm{CaCl}_{2}$ (YMS-MC medium) for sporulation. ISP medium 4 (Becton Dickinson) was used for intergenic conjugation from E. coli into Streptomyces. Spores $\left(7.0 \times 10^{8}\right.$ c.f.u. $)$ of S. avermitilis strains were inoculated into $70 \mathrm{ml}$ APM medium (Kitani et al., 2009) in a $500 \mathrm{ml}$ baffled flask, and mycelia were harvested after $48 \mathrm{~h}$ of cultivation. The mycelia were washed, resuspended in fresh APM medium and stored at $-80{ }^{\circ} \mathrm{C}$ until use as a seed culture. All the primers are listed in Supplementary Table S1.

Construction of the avaR3 deletion mutant. A segment upstream of avaR3 (nucleotides 4582958-4584926) was amplified by the primer pair avaR3-up-Fw/avaR3-up-Re, while a segment downstream of avaR3 (nucleotides 4580176-4582218) was amplified by the primer pair avaR3-dw-Fw/avaR3-dw-Re. The two resultant segments were digested with HindIII and SpeI, and inserted together into the HindIII site of pKU451, resulting in pLT341. The fidelity of the amplified region was confirmed by DNA sequencing. A $1.7 \mathrm{~kb}$ DNA fragment containing a kanamycin-resistant gene marker (amplified by the primer pair aph-Fw/aph-Re using pKU474 as template) was digested by SpeI, and cloned into the SpeI site of pLT341 to yield pLT342. A $5.7 \mathrm{~kb}$ HindIII fragment, recovered from pLT342, was transferred into pKU250 at the HindIII site, thereby yielding the avaR3-disruption plasmid pLT343. The resultant plasmid was introduced by intergenic conjugation into S. avermitilis KA320, and the wild-type gene was replaced with the disrupted allele by homologous recombination. The genotype of candidates for the avaR3 deletion was confirmed by PCR.

Complementation of the avaR3 mutant. A Gateway Reading Frame Cassette C.1 (Invitrogen) was cloned into the EcoRV site of pSET152 to yield pLT113 as a destination vector. A $1.2 \mathrm{~kb}$ DNA fragment (nucleotides 4581968-4583157) containing the entire avaR3 gene and the avaR3 upstream region was PCR-amplified by the primer pair avaR3-comp-Fw/avaR3-comp-Re, and then cloned into a pENTR vector according to the manufacturer's instructions to generate an entry clone. The entry clone was used with pLT113 in an LR reaction (LR Clonase Enzyme Mix, Invitrogen), resulting in pLT362. By intergenic conjugation, pLT362 was introduced into the avaR3 deletion mutant. The correct integration in the exconjugants was confirmed by PCR.

Analysis of antibiotic production. The seed culture was inoculated into $70 \mathrm{ml}$ APM medium in a $500 \mathrm{ml}$ baffled flask at a final $\mathrm{OD}_{600}$ of 0.3 , and cultivated at $28{ }^{\circ} \mathrm{C}$. The culture broth was sampled periodically, and mixed with an equal volume of methanol. After centrifugation, the supernatant was analysed by an HPLC system as described previously (Kitani et al., 2009). The amounts of four avermectin components, A1a, A2a, B1a and B2a, were quantified using an authentic sample of avermectins. For filipin III production, the supernatant was separated and quantified by reverse-phase HPLC 
(Mightysil RP-18GP column, $4.6 \times 250 \mathrm{~mm}$, Kanto Chemical) using $\mathrm{CH}_{3} \mathrm{CN} / \mathrm{MeOH} / \mathrm{H}_{2} \mathrm{O}(30: 20: 50$ by volume) as solvent and detection at $336 \mathrm{~nm}$. To measure antibiotic production on solid medium, spores $\left(1.0 \times 10^{5}\right.$ c.f.u. $)$ were inoculated on $2.5 \mathrm{ml}$ YMS-MC medium and APM agar medium (APM medium containing $1.5 \%$ agar), and incubated at $28{ }^{\circ} \mathrm{C}$ for 8 and 10 days, respectively. The culture was diced and extracted with methanol $(5 \mathrm{ml})$. The supernatant was collected by centrifugation and analysed by the HPLC system described above. The HPLC analysis of antibiotic production in liquid culture and on solid medium was performed at least twice independently.

Transcriptional analysis by RT-PCR. Total RNA was prepared from mycelium grown in APM medium by using an RNeasy Mini kit (Qiagen), and treated with DNase I (Takara Bio). The cDNA was synthesized using SuperScript III RNase $\mathrm{H}^{-}$Reverse Transcriptase (Invitrogen) and Random Primers (Invitrogen) according to the manufacturer's instructions. The PCR amplification was performed by using GoTaq Green Master Mix (Promega KK) under the following conditions: $97{ }^{\circ} \mathrm{C}$ for $3 \mathrm{~min}$, followed by discrete cycles (as described in the legend to Fig. 3) at $97{ }^{\circ} \mathrm{C}$ for $30 \mathrm{~s}, 58{ }^{\circ} \mathrm{C}$ for $30 \mathrm{~s}$, and $72{ }^{\circ} \mathrm{C}$ for $1 \mathrm{~min}$. The absence of DNA contamination was confirmed by RT-PCR without reverse transcriptase.

Quantitative RT-PCR (qRT-PCR) was performed using an Applied Biosystems 7300 Real-Time PCR system and SYBR Green PCR Master Mix (Applied Biosystems) according to the supplier's recommendations. The reaction parameters were as follows: $95{ }^{\circ} \mathrm{C}$ for $10 \mathrm{~min}$, followed by 40 cycles consisting of $15 \mathrm{~s}$ at $97{ }^{\circ} \mathrm{C}$ for denaturation, and $1 \mathrm{~min}$ at $65{ }^{\circ} \mathrm{C}$ for annealing and extension. A final dissociation stage was run to generate a melting curve and consequently verify the specificity of the amplification products. Gene expression was measured in triplicate and normalized to the mRNA level of the rpoD gene (sav2444) using the relative standard curve method.

Determination of transcriptional start points. A GeneRacer kit (Invitrogen) was used with total RNA extracted from $48 \mathrm{~h}$ mycelium for rapid amplification of $5^{\prime}$ cDNA ends (RACE) according to the manufacturer's instructions without dephosphorylation of RNA. The cDNA was synthesized using SuperScript III RNase $\mathrm{H}^{-}$Reverse Transcriptase and Random Primers. The first round of RACE-PCR was performed with a gene-specific reverse primer and the GeneRacer 5 ' Primer using GoTaq Green Master Mix. The second round of RACE-PCR was carried out with a gene-specific nested primer and the GeneRacer 5' Nested Primer using the initial PCR product as template. PCR products were cloned into a pCR4-TOPO vector (Invitrogen) for sequencing.

\section{RESULTS}

\section{Effects of avaR3 disruption on antibiotic production in $\mathbf{S}$. avermitilis}

A homology search with a FASTA program against the $S$. avermitilis genome demonstrated that there are three homologues of autoregulator receptor genes (sav3702, sav3703 and sav3705). The deduced gene product of sav3705, designated avaR1 ( $S$. avermitilis autoregulator receptor gene 1 ), showed the highest sequence similarity (39-48\% identity) to $\gamma$-butyrolactone autoregulator receptors, such as ArpA, BarA and ScbR. Notably, two homologue genes (sav3702 and sav3703), whose products showed moderate similarity (25-34\% identity) to the autoregulator receptors but much higher similarity (36$48 \%$ identity) to receptor homologues (BarB and ScbR2), were found to be present in the adjacent region of the avaR1 gene (Fig. 1a). Thus, these genes were designated avaR2 and avaR3, respectively. Sequence alignment with known autoregulator receptors (Fig. 1b) revealed that the predicted products of the three avaR genes had two features: a highly conserved helix-turnhelix (HTH) DNA-binding domain at the $\mathrm{N}$ terminus (Pfam00440), and a conserved tryptophan residue at the centre of proteins, which is proposed to be an important amino acid residue for the function of the autoregulator receptor in responding to the binding of the $\gamma$ butyrolactone autoregulator (Natsume et al., 2004). These findings indicated that genes encoding homologues of autoregulator receptors are clustered at the same locus in S. avermitilis (nucleotides $4581428-4585275$ ), suggesting that they are probably involved in the regulation of secondary metabolism and/or morphological differentiation.

Unlike typical domain structures of the $\gamma$-butyrolactone autoregulator receptor, the predicted AvaR3 protein has an extra stretch of 75 aa residues between the HTH DNAbinding domain and the conserved tryptophan residue (Fig. 1b). Such an extra stretch is very rare and only known in AlpW (stretch of 24 aa residues) of Streptomyces ambofaciens among the autoregulator receptors (Bunet et al., 2011). A search of the current database demonstrated that there is no protein that contains the stretch possessed by AvaR3. The nucleotide sequence encoding the 75 aa stretch had a relatively low $\mathrm{G}+\mathrm{C}$ content at the third letter of the codon, i.e. $73 \%$ versus an average of $92 \%$ in Streptomyces genes (Ishikawa \& Hotta, 1999). The avaR3 gene was constitutively expressed throughout cultivation (Fig. 1c), suggesting that AvaR3 may have a physiological function. In order to investigate the in vivo role of avaR3 in S. avermitilis, an avaR3 deletion ( $\triangle a v a R 3)$ mutant was constructed by allelic replacement. With respect to antibiotic production, avermectin production of the wild-type strain was readily detectable after $72 \mathrm{~h}$ of cultivation and increased until $120 \mathrm{~h}$ of cultivation, whereas the $\triangle a v a R 3$ mutant produced trace amounts of avermectins (less than $1 \%$ of the amount produced by the wild-type strain) (Fig. 2a). In contrast, the $\Delta a v a R 3$ mutant showed a sevenfold increase in production of filipin III at $72 \mathrm{~h}$ of cultivation in comparison with that of the wildtype strain (Fig. 2b). With respect to production of oligomycin $\mathrm{A}$, there was no difference between the wildtype strain and the $\triangle a v a R 3$ mutant (data not shown). Complementation of the $\triangle a v a R 3$ mutant, with the genome-integrative plasmid pLT362 containing the entire avaR3 gene and its upstream region, restored the production of avermectins and filipin III to the level of the wild-type strain (Fig. 2a, b). These results clearly indicated that AvaR3 functions as a positive regulator of avermectin production and has a negative effect on filipin production. 
(a)

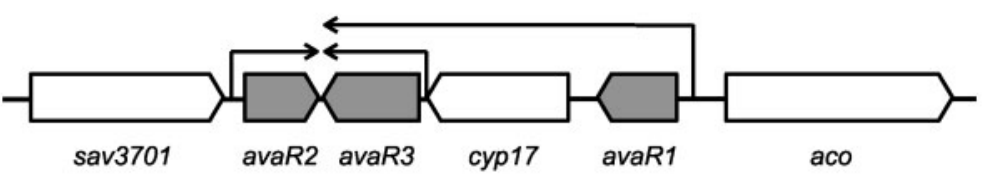

(c)

\begin{tabular}{llllll}
\multicolumn{7}{c}{ Time (h) } \\
\hline 12 & 24 & 36 & 48 & 60 & 72 \\
\hline & & & & & \\
\hline & & & & & \\
\hline
\end{tabular}

(b)
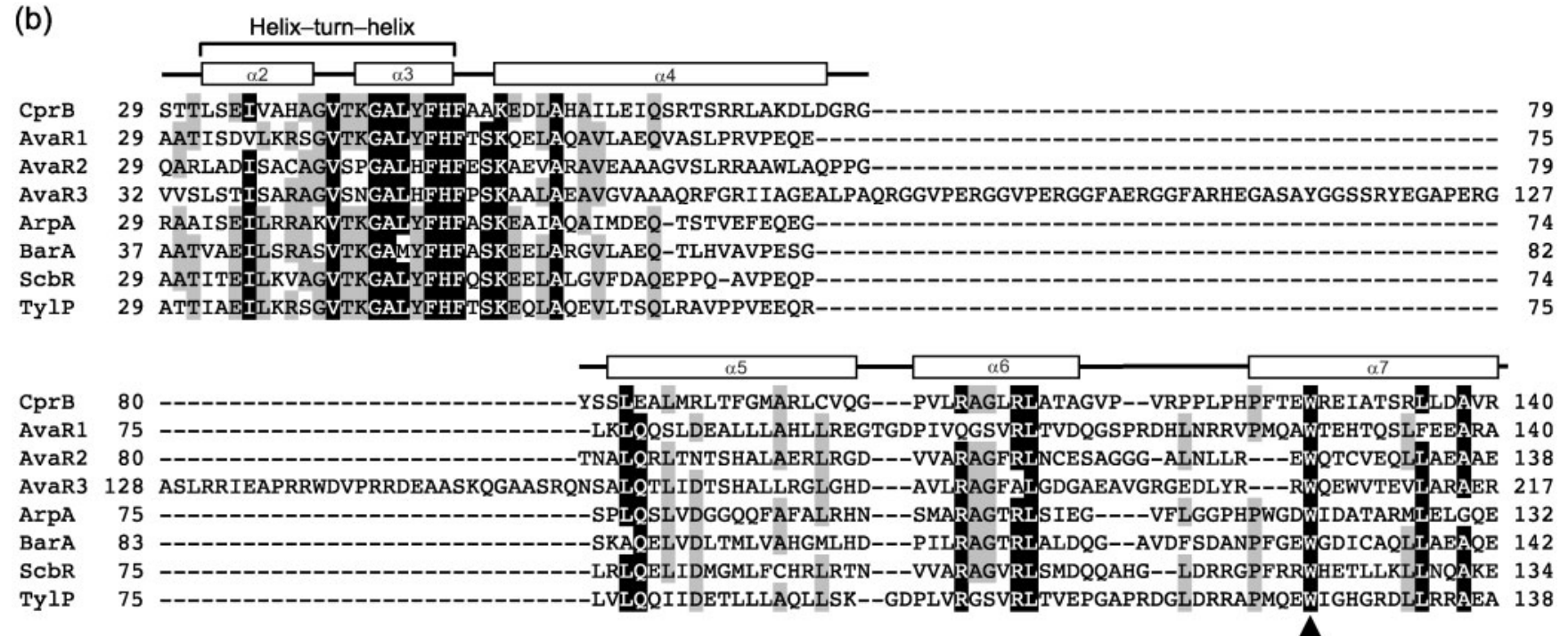

Fig. 1. Organization of the avaR genes in S. avermitilis at nucleotides 4581428 to 4585275 (a), sequence alignment of the $\mathrm{N}$-terminal regions of AvaR proteins with those of $\gamma$-butyrolactone autoregulator receptors (b), and transcriptional analysis of the avaR3 gene in the wild-type strain (c). (a) Bent arrows indicate transcriptional units of the avaR genes. (b) The amino acid sequence alignment was created using CLUSTAL W. The numbers indicate the amino acid positions within each sequence. The secondary structure elements of $\mathrm{CprB}$ are shown above its sequence. The filled triangle indicates an important residue for conformational changes of the autoregulator receptor upon binding of the $\gamma$-butyrolactone autoregulator, as proposed by Natsume et al. (2004). Black and grey boxes in the alignment indicate positions at which the same amino acid is found in at least seven and five of the eight sequences, respectively. CprB (BAA28748) and ScbR (CAA07628) from S. coelicolor A3(2); AvaR1 (BAC71417), AvaR2 (BAC71414) and AvaR3 (BAC71415) from S. avermitilis; ArpA (BAA36282) from S. griseus; BarA (BAA06981) from S. virginiae; TyIP (AAD40801) from Streptomyces fradiae. (c) Total RNA was prepared from mycelium at the indicated cultivation times. PCR with 25 cycles of amplification was used for transcripts of the avaR3 and rpoD genes. The $r p o D$ gene was used as a control.

\section{Influence of AvaR3 on the transcription of avermectin biosynthetic genes}

To elucidate the function of avaR3 in transcriptional regulation of antibiotic biosynthetic genes, the pattern of gene expression was examined by RT-PCR analysis (Fig. 3). All of the investigated avermectin biosynthetic genes were found to be transcribed in the wild-type strain at $48 \mathrm{~h}$ of cultivation, and avermectin production began several hours after the $48 \mathrm{~h}$ cultivation time point. However, in the $\triangle a v a R 3$ mutant, the expression at $48 \mathrm{~h}$ of five biosynthetic genes, aveA1, aveA4, aveBI, aveBIII and aveBVIII, still remained at low levels similar to those observed at $24 \mathrm{~h}$ of cultivation, while the other three genes, aveD, aveBIV and ave $G$, were transcribed normally. At $72 \mathrm{~h}$ of cultivation, the transcriptional levels of these five genes were elevated. The avaR3-complemented exconjugant showed the same temporal expression pattern of the avermectin biosynthetic genes as the wild-type strain, suggesting that AvaR3 upregulates the expression of a part of the ave cluster. AveR positively controls the expression of both polyketide biosynthetic and post-polyketide modification genes in avermectin biosynthesis (Kitani et al., 2009). Reduced amounts of aveR transcripts were also detected throughout the cultivation of the $\Delta a v a R 3$ mutant, in contrast to the results with the wild-type strain. This result indicated that AvaR3 is necessary to expedite the transcription of aveR, implying that the remarkable decrease of avermectin production is due to a lower and delayed transcription of the avermectin biosynthetic genes regulated by aveR. Surprisingly, major filipin biosynthetic genes (pte), including the putative pathway-specific regulatory genes $p t e F$ and $p t e R$, were transcribed in the $\Delta a v a R 3$ mutant in a manner similar to that of the wild-type strain (Supplementary Fig. 

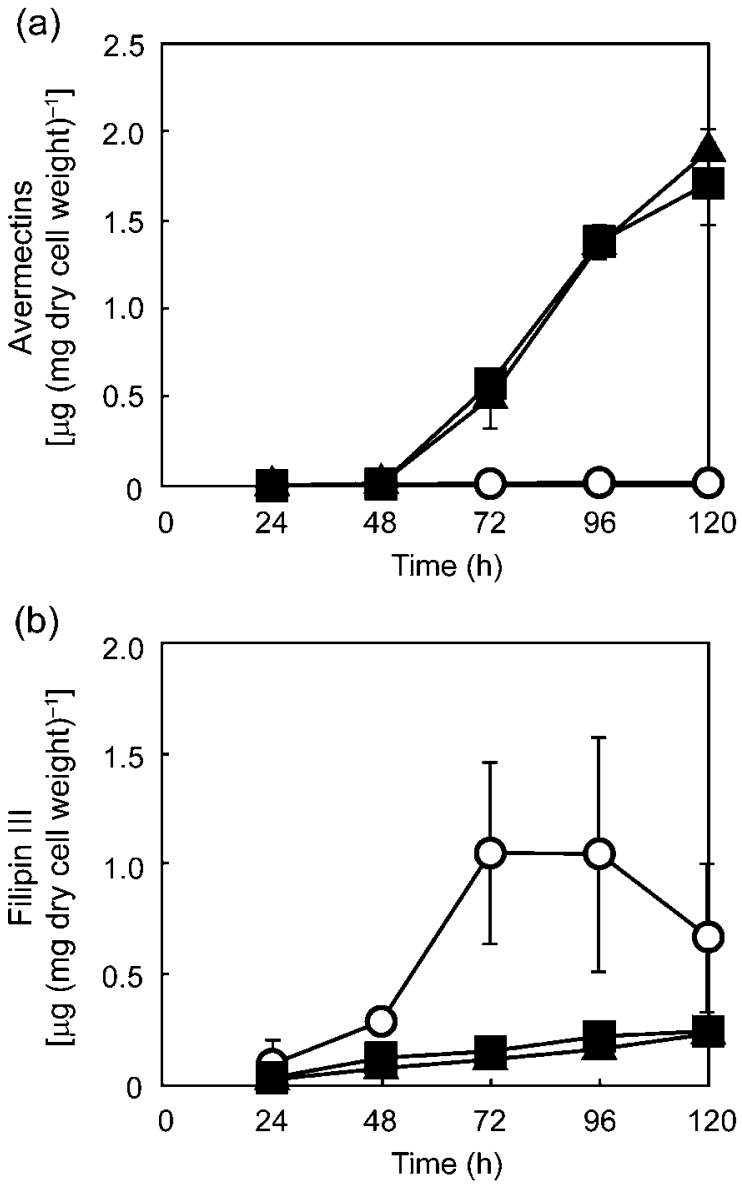

Fig. 2. Production profiles of avermectin (a) and filipin (b) in the wild-type strain ( $\mathbf{\square}), \Delta$ avaR3 mutant $(\bigcirc)$ and avaR3-complemented exconjugant $(\boldsymbol{\Delta})$ in liquid culture. Error bars, SD from triplicate cultures.

S2), in spite of the overproduction of filipin in the $\triangle a v a R 3$ mutant. This observation suggested that AvaR3 has a negative effect on the production of filipin without transcriptional control of the pte gene cluster.

\section{Antibiotic production of the $\Delta a v a R 3$ mutant on solid medium}

To investigate the contribution of avaR3 to the regulation of antibiotic production on solid medium, we assessed the production of avermectin, filipin and oligomycin on APM agar medium and YMS-MC medium (Table 1). In the cultivation on APM agar medium, the wild-type strain produced $74 \mu$ g avermectins $(\mathrm{ml} \text { medium })^{-1}$, whereas the $\triangle a v a R 3$ mutant showed a $79 \%$ reduction $\left(16 \mu \mathrm{g} \mathrm{ml}^{-1}\right)$ in avermectin production. Meanwhile, filipin production of the $\triangle a v a R 3$ mutant increased to a level 3.3-fold greater than that of the wild-type strain. No differences in oligomycin production were detected between the wildtype strain and the $\triangle a v a R 3$ mutant (data not shown). These phenotypic changes attributable to the avaR3

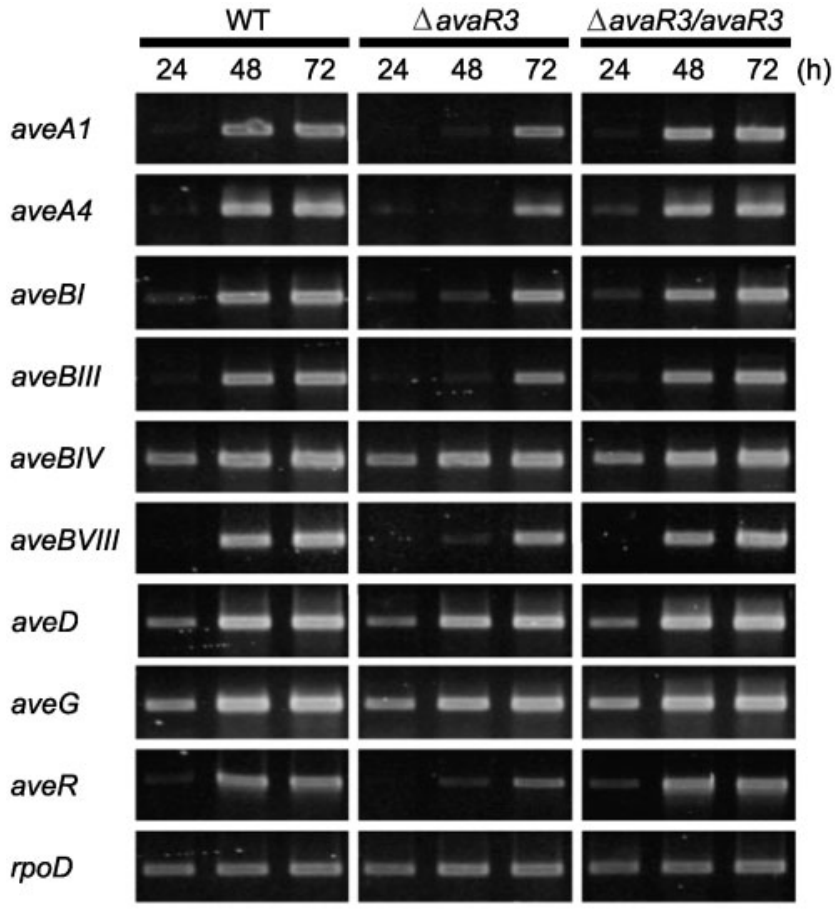

Fig. 3. Gene expression analysis by RT-PCR of avermectin biosynthetic genes in the wild-type strain (WT), $\Delta$ avaR3 mutant ( $\triangle a v a R 3)$ and avaR3-complemented exconjugant ( $\triangle a v a R 3 /$ avaR3). Total RNA was isolated from mycelia harvested at 24, 48 and $72 \mathrm{~h}$ of cultivation. Avermectin production started between 48 and $72 \mathrm{~h}$ of cultivation. In the PCR, the following numbers of amplification cycles were used: 27 cycles for the ave $R$ and aveBVIII transcripts, 25 cycles for the $r p o D$ transcript, and 30 cycles for the transcripts of the other genes.

mutation were partially restored by introduction of the intact avaR3 gene. Similarly, cultivation of the $\triangle a v a R 3$ mutant on YMS-MC medium resulted in a $69 \%$ decrease of avermectin production and a 1.7 -fold increase of filipin production with no change in oligomycin production. Under these medium conditions, the changes in avermectin and filipin production were restored to the wild-type level by the introduction of the intact avaR3 gene. These phenomena on solid medium were similar to the antibiotic production in liquid cultivation, indicating that AvaR3 is also involved in the production of avermectins and filipin III on solid medium.

\section{avaR3 mutation affects cell morphology on solid culture and in liquid culture}

Some $\gamma$-butyrolactone autoregulator receptors control both morphological differentiation and antibiotic production (Folcher et al., 2001; Healy et al., 2009; Horinouchi, 2007). To clarify whether AvaR3 is involved in the morphological control of S. avermitilis, we carefully examined morphological characteristics of the $\triangle a v a R 3$ mutant on a variety of solid media. On YMS-MC medium, where the $\Delta a v a R 3$ 
Table 1. Antibiotic production of the $S$. avermitilis wild-type strain, a $\Delta a v a R 3$ mutant and an avaR3-complemented exconjugant on solid media

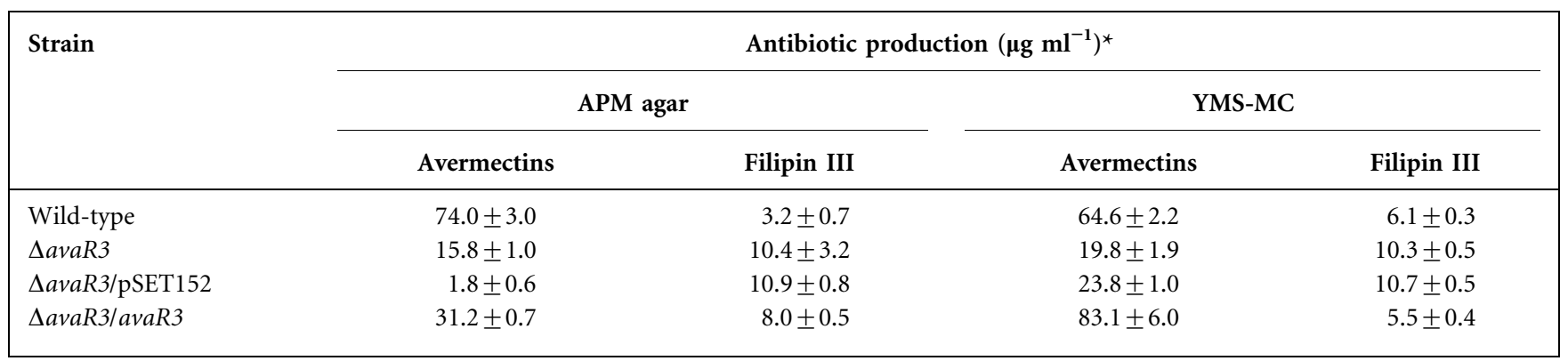

${ }^{*}$ Data shown are mean \pm SD from triplicate samples.

mutant showed less avermectin production and more filipin production, no differences in morphology were detected between the wild-type strain and the $\triangle a v a R 3$ mutant. On APM agar medium, however, the $\triangle a v a R 3$ mutant showed a clear defect in aerial mycelium formation at the time point at which the wild-type strain produced an abundant lawn of aerial mycelium, although the $\triangle a v a R 3$ mutant grew normally until the stage of substrate mycelium (Supplementary Fig. S3), suggesting that the process of aerial mycelium formation is most likely impaired in the $\triangle a v a R 3$ mutant. To determine the effect of avaR3 disruption on development more quantitatively, the degree of sporulation was investigated by counting a number of spores. Fig. 4(a) demonstrates that the number of spores in the $\triangle a v a R 3$ mutant was 5.8-fold lower than that of the wild-type strain, indicating the clear involvement of AvaR3 in morphological development. Complementation by intact avaR3 restored sporulation, but only partially (35\% of the wild-type strain). We suspected that integration at the $a t t B$ site might have an adverse effect. In fact, integration of an empty vector alone ( $\triangle a v a R 3 / p S E T 152 ;$ Fig. 4a) impaired sporulation severely. A similar adverse effect due to $a t t B$ site integration was also observed on avermectin production (Table 1) when the mutant strain was cultured on APM agar medium alone, suggesting that avaR3 and the attB site adjacent gene(s) may have a similar level of importance in antibiotic production and morphological development, when grown on APM agar medium.

In liquid culture, the avaR3 mutation showed a 1.7- to 1.9fold increase in the $\mathrm{OD}_{600}$ value after 48 to $72 \mathrm{~h}$ cultivation, respectively (data not shown), although the dry cell weight of the $\triangle a v a R 3$ mutant was lower than that of the wild-type strain after $72 \mathrm{~h}$ of cultivation (Fig. $4 \mathrm{~b}$ ). Micrographs (Fig. 4c) revealed that the $\triangle a v a R 3$ mutant possessed more fragmented mycelia, with a remarkable decrease in the mean diameter of the pellet $(73 \pm 6 \mu \mathrm{m})$, while the wild-type strain produced typical large pellets with a mean diameter of $154 \pm 14 \mu \mathrm{m}$, indicating that the increased $\mathrm{OD}_{600}$ value of the $\triangle$ avaR3 mutant would have resulted from the increased dispersion due to the decreased pellet size during cultivation, and that AvaR3 may play an important role in mycelial aggregation in liquid culture.

\section{Transcriptional start points of the avaR genes}

ARE sequences, to which $\gamma$-butyrolactone autoregulator receptor proteins typically bind, are frequently found in the promoter region of their own genes as well as those of the direct target genes (Folcher et al., 2001). To analyse the promoter region of avaR3, 5'-RACE analysis was carried out (Supplementary Fig. S4), which revealed that the transcriptional start point (tsp) of avaR3 was A, situated $25 \mathrm{nt}$ upstream from the translational start codon of avaR3 (Fig. 5a). In front of the tsp, a possible -10 region was identified, which was similar to the consensus -10 region of streptomycetes $\mathrm{E} \sigma^{70}$-like promoters, although no typical -35 region was detected (Kang et al., 1997; Strohl, 1992). As shown in Fig. 5(a), an ARE-like sequence was found at -36 to $-11 \mathrm{nt}$ from the tsp of avaR3, and designated avaR3-ARE.

Similarly, avaR1-tsp was located at G, $102 \mathrm{nt}$ upstream of the translational start codon, and avaR2-tsp at T, $43 \mathrm{nt}$ upstream of the translational start codon (Fig. 5b, c and Supplementary Fig S4), and both possessed probable promoters, resembling the consensus -10 region of streptomycetes $\mathrm{E} \sigma^{70}$-like promoters (avaR1), or -10 and -35 regions of the $\sigma^{h r d D}$-type promoters of $S$. coelicolor A3(2) (avaR2). As shown in Fig. 5(b, c), ARE-like sequences were identified in the upstream regions of these two genes as avaR1-ARE, which was situated at -93 to $-68 \mathrm{nt}$ from the avaR1-tsp, and as avaR2-ARE, which covers both the avaR2tsp and the putative -10 region.

Regarding the gene organization, avaR3 runs in the same direction as the upstream avaR1 and cyp17 (sav3704) (Fig. 1a), which encodes a cytochrome P450 hydroxylase, CYP154B2 (Lamb et al., 2003). Because no plausible transcriptional terminator exists in the $3^{\prime}$ regions of cyp 17 and avaR1, avaR3 may form a tricistronic operon with cyp17 and avaR1. RT-PCR analysis using RNA from the $48 \mathrm{~h}$ mycelia (Supplementary Fig. S5) detected transcripts containing the intergenic regions of cyp17-avaR3 and 

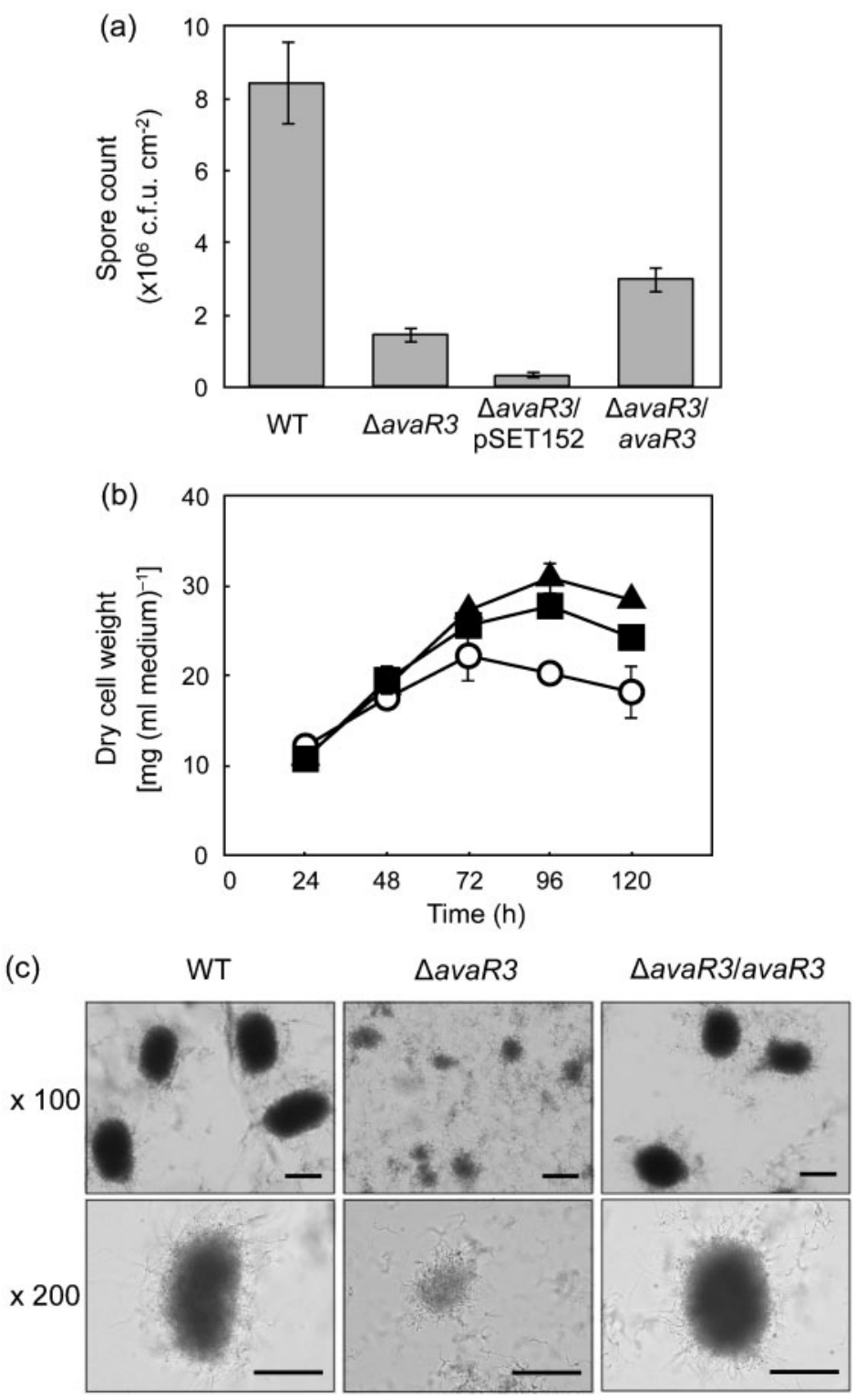

Fig. 4. Effect of avaR3 deletion on cell morphology on agar medium and in liquid medium. All experiments were conducted at least twice, and the results were highly reproducible. WT, wild-type strain; $\triangle a v a R 3$, $\triangle a v a R 3$ mutant; $\triangle a v a R 3 / p S E T 152, \Delta a v a R 3$ carrying pSET152; $\Delta a v a R 3 / a v a R 3$, avaR3complemented exconjugant. Error bars, SD from triplicate cultures (a, b). (a) Spore numbers on agar medium. The spores $\left(1.0 \times 10^{7}\right.$ c.f.u. $)$ of each strain were plated on $25 \mathrm{ml}$ APM agar medium, followed by incubation for 10 days at $28^{\circ} \mathrm{C}$. (b) Growth curve in liquid culture measured by dry cell weight: wild-type strain ( $\mathbf{\square}), \Delta$ avaR3 mutant $(\bigcirc)$ and avaR3-complemented exconjugant (A). (c) Phase-contrast micrographs of the mycelia in liquid medium. Cultures were grown in APM medium for $72 \mathrm{~h}$. The micrographs were taken with an Olympus BX50 microscope and an Olympus DP70 digital camera. Bars, $100 \mu \mathrm{m}$. The diameter was measured from 30 independent pellets, and calculated as mean and SD.

avaR1-cyp17, and the tricistronic transcript. This finding, together with the fact that 5 '-RACE analysis did not yield any distinct bands of the cyp 17 transcript, led us to conclude that avaR3 is transcribed as a monocistronic mRNA and a tricistronic avaR1-cyp17-avaR3 mRNA. The presence of ARE in all three avaR genes suggested that AvaR3 might transcriptionally control the expression of itself, avaR 1 and avaR2 via binding to AREs.

\section{Transcriptional control by AvaR3 of the avaR genes}

To examine whether AvaR3 regulates the transcription of the avaR genes, qRT-PCR analysis was performed as shown in Fig. 6. The mRNA level of avaR3 increased significantly in the $\triangle a v a R 3$ mutant compared with that in the wild-type strain (2.8- to 4.8-fold), indicating that an autoregulatory circuit is present in avaR3 transcription. Increased mRNA levels of avaR1 and avaR2 in the $\triangle a v a R 3$ mutant led us to conclude that AvaR3 also negatively regulates the transcription of both avaR1 and avaR2. These results indicated that all the avaR genes are under the control of AvaR3. avaR1 disruption did not give any of the phenotypic changes observed in the $\triangle a v a R 3$ mutant (Supplementary Fig. S6), indicating that AvaR3 controls antibiotic production as well as cell morphology independently of AvaR1. Further work will be needed to find out whether AvaR2 participates in AvaR3-dependent phenomena.

\section{DISCUSSION}

The $\gamma$-butyrolactone autoregulator receptor and its homologues are distributed widely among streptomycetes, and control antibiotic production and/or morphological 
(a) CCCTGCCGGTCGTCCTGCGGCCGCACTGATGCGCGAGAAAACCGCACAAGCG

$\begin{array}{lllllllllllll}\text { L } & P & V & V & \text { L } & R & P & H & \text { * }\end{array}$

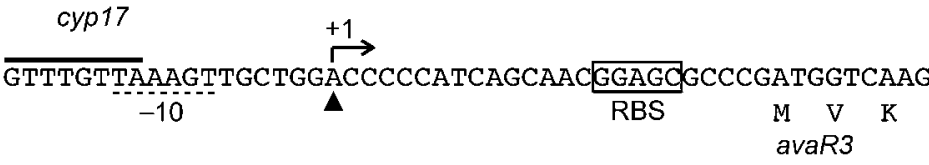

(b) GCATTGAAAAACCGTTCAGCTGGTTTTATCGTCCCGGTCAGGACCGGGCCTG TCTTCGGCGGTGGAGTGGAGGATCGTCGCTGTTAGGGTGCGAACCGCTTGGT CTTTTCCCGCTGGTCGGGACGGTGTCCCGCGATCCGGACGGTGTCCCACTGG TCGGGACGGTGTCCCGCTGGTCGGGCTGGTGCGGAGGGTGCGTGTGGCGCGG RBS M A R avaR1

(c)

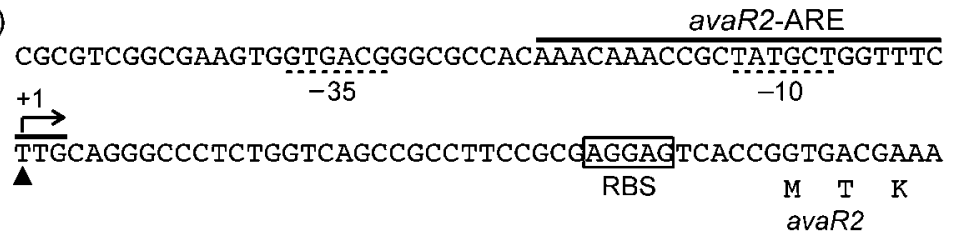

Fig. 5. Nucleotide sequences of the upstream regions of avaR3 (a), avaR1 (b) and avaR2 (c). The solid triangle indicates the transcriptional start point. The probable -35 and -10 regions are shown by dashed lines, and the putative ribosome-binding sites (RBSs) are shown in boxes. The putative AvaR3-binding sequences are indicated by solid lines. differentiation. In this study, we have characterized AvaR3, an autoregulator receptor homologue in S. avermitilis, and have shown that it is responsible for the production of avermectin and filipin, and for the control of cell morphology, both in liquid medium and on solid medium. These findings led to the idea that AvaR3 is an activator-type global regulator for secondary metabolism and morphogenesis, except for filipin production (see Discussion below). In S. griseus, ArpA stimulates the onset of secondary metabolism and morphological differentiation in response to Afactor through transcriptional regulation of AdpA, a central transcriptional activator. Furthermore, a few AdpA homo-

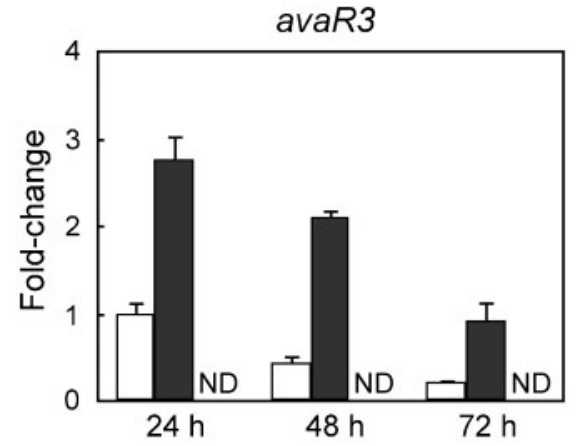

WT
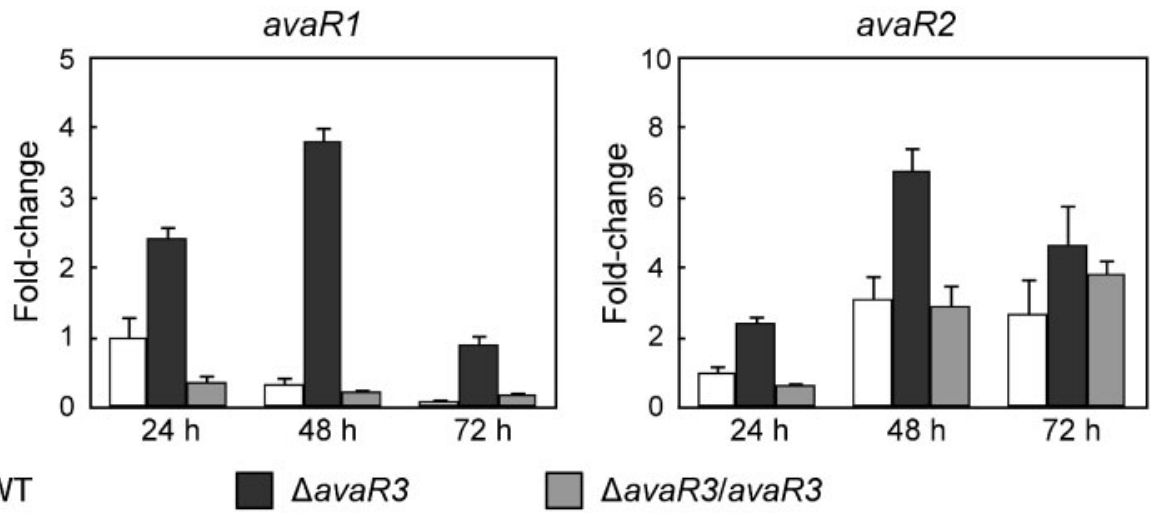

Fig. 6. Gene expression analysis of the avaR3, avaR1 and avaR2 genes by qRT-PCR. WT, wild-type strain; $\Delta a v a R 3, \Delta a v a R 3$ mutant; $\triangle a v a R 3 / a v a R 3$, avaR3-complemented exconjugant. Total RNA was extracted from mycelia harvested at the indicated cultivation times. Fold-change is relative to the expression of each gene in the wild-type strain at $24 \mathrm{~h}$ of cultivation. The avaR3 transcript corresponding to +1 to $+58 \mathrm{nt}$ from the transcriptional start point of avaR3 was quantified. ND, The mRNA level of avaR3 was not determined in the avaR3-complemented strain, because the amplified regions upstream of avaR3 are present at two loci. Error bars, SD from triplicate experiments. 
logues in other Streptomyces strains have been shown to be pleiotropic regulators for secondary metabolism and morphological differentiation (López-García et al., 2010; Pan et al., 2009). However, the transcription of $b d p A$ (sav5261), a plausible adpA orthologue of $S$. avermitilis, is not influenced by the avaR3 disruption (data not shown). Thus, this finding indicates that AvaR3 has a $b d p A$ independent pathway for controlling avermectin production and cell morphology, suggesting that $S$. avermitilis has a novel regulatory system via autoregulator receptors that globally regulates two representative features of streptomycetes.

In the usual pattern of antibiotic regulation, a gene encoding a $\gamma$-butyrolactone autoregulator receptor resides inside or adjacent to an antibiotic biosynthetic gene cluster and exerts a negative effect on antibiotic production by binding of the receptor protein to the promoter region of the in-cluster pathway-specific regulatory gene (Bunet et al., 2008; Cundliffe, 2008). It was thus of interest that AvaR3 regulates the production of avermectin, whose biosynthetic gene cluster is $3.4 \mathrm{Mb}$ away from the avaR3 locus. Moreover, although AvaR3 facilitates the expression of aveR, a pathway-specific regulatory gene for avermectin biosynthesis, no plausible ARE sequence is located in the region upstream of the aveR gene, suggesting that AvaR3 is likely to control the transcription of aveR without direct binding to the promoter.

In spite of the increased expression of avermectin biosynthetic genes at the late growth stage, the $\triangle a v a R 3$ mutant still showed little production of avermectin and increased filipin production with no change of transcription of filipin biosynthetic genes. Because the biosynthetic pathways of both avermectin and filipin require malonyl-CoA and methylmalonyl-CoA as a common extender unit of the polyketide backbone (Ikeda et al., 1999; Omura et al., 2001), it is most likely that the delayed expression of the ave genes, attributed to the delayed and weakened transcription of aveR as the result of avaR3 disruption, would lead to the preferential use of precursors in filipin production at the middle growth stage, resulting in increased filipin production and a lack of the necessary precursors for avermectin production at the late growth stage. Regarding oligomycin production, although oligomycin biosynthesis shares common precursors (malonyl-CoA and methylmalonyl-CoA) with avermectin and filipin biosynthesis (Wei et al., 2006), because oligomycin production already reached a plateau before the onset of avermectin production in the wild-type strain (Supplementary Fig. S6), the surplus precursors resulting from the delayed expression of ave genes in the $\Delta a v a R 3$ mutant had no influence on oligomycin production. Thus, AvaR3 appears to act as a transcriptional activator for avermectin production by indirectly controlling the expression of aveR.

To date, the molecular mechanisms underlying mycelial aggregation in liquid cultivation remain poorly understood. Recently, two factors have been reported to be involved in the process of mycelial aggregation: SsgA, which is an activator of sporulation-specific cell division (Traag \& van Wezel, 2008), and CslA, which is involved in polysaccharide synthesis at the outer surface of hyphae (de Jong et al., 2009). The enhanced expression of $s s g A$ leads to mycelial fragmentation in $S$. coelicolor A3(2) and S. griseus (Kawamoto et al., 1997; van Wezel et al., 2000), whereas the cslA mutation reduces the degree of mycelial aggregation in S. coelicolor A3(2) (Xu et al., 2008). We have demonstrated in this study that the avaR3 mutation results in fragmented growth in liquid culture. Putative genes encoding the SsgA and CslA orthologues are present in the genome of S. avermitilis as SAV_4267 and SAV_5219, respectively. The transcriptional profile of these two genes in the $\triangle a v a R 3$ mutant was nearly identical to that of the wild-type strain (data not shown), suggesting that AvaR3 regulates mycelial aggregation independently of an SsgA- and CslA-dependent pathway. To the best of our knowledge, this is the first report to note that an autoregulator receptor homologue participates in both mycelial clump formation in liquid culture and aerial mycelium formation on solid medium.

The completed Streptomyces genome sequences have demonstrated that the putative receptor genes are frequently far from the antibiotic biosynthetic gene clusters, although the majority of reports on autoregulator receptors have so far concentrated on their pathway-specific functioning. Thus, it is expected that further understanding of the regulatory mechanism of AvaR3 will lead not only to higher yields in antibiotic production but also to the ability to awaken silent gene clusters for the discovery of novel natural compounds.

\section{ACKNOWLEDGEMENTS}

This work is a part of the $\mathrm{PhD}$ dissertation of K.T.M. We thank Hiroshi Kinoshita for his helpful suggestions, and Atsushi Okazawa for photography assistance. We also thank Satoshi Harashima, Minetaka Sugiyama and Walter A. Lavinia for assistance with qRTPCR analysis. This work was supported by a research grant from the Noda Institute for Scientific Research (to S. K.), by a grant for the Joint Program in the Field of Biotechnology under the Japan Society for the Promotion of Science, the National Research Council of Thailand and National Science and Technology Development Agency of Thailand (to T. N.), by a Grant-in-Aid for Scientific Research (B) (no. 21360404) from the Ministry of Education, Culture, Sports, Science and Technology (MEXT) of Japan (to T.N. and S. K.), by a Grant-in-Aid for Scientific Research on Innovative Areas (no. 20310122) from MEXT, and by a grant from the Institute for Fermentation, Osaka, Japan (to H. I.).

\section{REFERENCES}

Bibb, M. J. (2005). Regulation of secondary metabolism in streptomycetes. Curr Opin Microbiol 8, 208-215.

Bierman, M., Logan, R., O’Brien, K., Seno, E. T., Rao, R. N. \& Schoner, B. E. (1992). Plasmid cloning vectors for the conjugal transfer of DNA from Escherichia coli to Streptomyces spp. Gene 116, 43-49.

Bunet, R., Mendes, M. V., Rouhier, N., Pang, X., Hotel, L., Leblond, P. \& Aigle, B. (2008). Regulation of the synthesis of the angucyclinone antibiotic alpomycin in Streptomyces ambofaciens by the autoregulator receptor AlpZ and its specific ligand. J Bacteriol 190, 3293-3305. 
Bunet, R., Song, L., Mendes, M. V., Corre, C., Hotel, L., Rouhier, N., Framboisier, X., Leblond, P., Challis, G. L. \& Aigle, B. (2011). Characterization and manipulation of the pathway-specific late regulator AlpW reveals Streptomyces ambofaciens as a new producer of kinamycins. J Bacteriol 193, 1142-1153.

Burg, R. W., Miller, B. M., Baker, E. E., Birnbaum, J., Currie, S. A., Hartman, R., Kong, Y. L., Monaghan, R. L., Olson, G. \& other authors (1979). Avermectins, new family of potent anthelmintic agents: producing organism and fermentation. Antimicrob Agents Chemother 15, 361-367.

Cundliffe, E. (2008). Control of tylosin biosynthesis in Streptomyces fradiae. J Microbiol Biotechnol 18, 1485-1491.

de Jong, W., Wösten, H. A., Dijkhuizen, L. \& Claessen, D. (2009). Attachment of Streptomyces coelicolor is mediated by amyloidal fimbriae that are anchored to the cell surface via cellulose. Mol Microbiol 73, 1128-1140.

Folcher, M., Gaillard, H., Nguyen, L. T., Nguyen, K. T., Lacroix, P., Bamas-Jacques, N., Rinkel, M. \& Thompson, C. J. (2001). Pleiotropic functions of a Streptomyces pristinaespiralis autoregulator receptor in development, antibiotic biosynthesis, and expression of a superoxide dismutase. J Biol Chem 276, 44297-44306.

Gottelt, M., Kol, S., Gomez-Escribano, J. P., Bibb, M. \& Takano, E. (2010). Deletion of a regulatory gene within the cpk gene cluster reveals novel antibacterial activity in Streptomyces coelicolor A3(2). Microbiology 156, 2343-2353.

Healy, F. G., Eaton, K. P., Limsirichai, P., Aldrich, J. F., Plowman, A. K. \& King, R. R. (2009). Characterization of $\gamma$-butyrolactone autoregulatory signaling gene homologs in the angucyclinone polyketide WS5995B producer Streptomyces acidiscabies. J Bacteriol 191, 4786-4797.

Horinouchi, S. (2007). Mining and polishing of the treasure trove in the bacterial genus Streptomyces. Biosci Biotechnol Biochem 71, 283-299.

Ikeda, H., Kotaki, H. \& Omura, S. (1987). Genetic studies of avermectin biosynthesis in Streptomyces avermitilis. J Bacteriol 169, 5615-5621.

Ikeda, H., Nonomiya, T., Usami, M., Ohta, T. \& Omura, S. (1999). Organization of the biosynthetic gene cluster for the polyketide anthelmintic macrolide avermectin in Streptomyces avermitilis. Proc Natl Acad Sci U S A 96, 9509-9514.

Ishikawa, J. \& Hotta, K. (1999). FramePlot: a new implementation of the frame analysis for predicting protein-coding regions in bacterial DNA with a high G + C content. FEMS Microbiol Lett 174, 251-253.

Kang, J. G., Hahn, M. Y., Ishihama, A. \& Roe, J. H. (1997). Identification of sigma factors for growth phase-related promoter selectivity of RNA polymerases from Streptomyces coelicolor A3(2). Nucleic Acids Res 25, 2566-2573.

Kawamoto, S., Watanabe, H., Hesketh, A., Ensign, J. C. \& Ochi, K. (1997). Expression analysis of the ssgA gene product, associated with sporulation and cell division in Streptomyces griseus. Microbiology 143, 1077-1086.

Kieser, T., Bibb, M. J., Buttner, M. J., Chater, K. F. \& Hopwood, D. A. (2000). Practical Streptomyces Genetics. Norwich: John Innes Foundation.

Kinoshita, H., Ipposhi, H., Okamoto, S., Nakano, H., Nihira, T. \& Yamada, Y. (1997). Butyrolactone autoregulator receptor protein (BarA) as a transcriptional regulator in Streptomyces virginiae. J Bacteriol 179, 6986-6993.

Kitani, S., Ikeda, H., Sakamoto, T., Noguchi, S. \& Nihira, T. (2009). Characterization of a regulatory gene, aveR, for the biosynthesis of avermectin in Streptomyces avermitilis. Appl Microbiol Biotechnol 82, 1089-1096.

Komatsu, M., Uchiyama, T., Omura, S., Cane, D. E. \& Ikeda, H. (2010). Genome-minimized Streptomyces host for the heterologous expression of secondary metabolism. Proc Natl Acad Sci U S A 107, 2646-2651.
Lamb, D. C., Ikeda, H., Nelson, D. R., Ishikawa, J., Skaug, T., Jackson, C., Omura, S., Waterman, M. R. \& Kelly, S. L. (2003). Cytochrome p450 complement (CYPome) of the avermectin-producer Streptomyces avermitilis and comparison to that of Streptomyces coelicolor A3(2). Biochem Biophys Res Commun 307, 610-619.

López-Garcia, M. T., Santamarta, I. \& Liras, P. (2010). Morphological differentiation and clavulanic acid formation are affected in a Streptomyces clavuligerus adpA-deleted mutant. Microbiology 156, 2354-2365.

Matsuno, K., Yamada, Y., Lee, C. K. \& Nihira, T. (2004). Identification by gene deletion analysis of $\operatorname{bar} B$ as a negative regulator controlling an early process of virginiamycin biosynthesis in Streptomyces virginiae. Arch Microbiol 181, 52-59.

Nakano, H., Lee, C. K., Nihira, T. \& Yamada, Y. (2000). A null mutant of the Streptomyces virginiae barA gene encoding a butyrolactone autoregulator receptor and its phenotypic and transcriptional analysis. J Biosci Bioeng 90, 204-207.

Natsume, R., Ohnishi, Y., Senda, T. \& Horinouchi, S. (2004). Crystal structure of a $\gamma$-butyrolactone autoregulator receptor protein in Streptomyces coelicolor A3(2). J Mol Biol 336, 409-419.

Omura, S., Ikeda, H., Ishikawa, J., Hanamoto, A., Takahashi, C., Shinose, M., Takahashi, Y., Horikawa, H., Nakazawa, H. \& other authors (2001). Genome sequence of an industrial microorganism Streptomyces avermitilis: deducing the ability of producing secondary metabolites. Proc Natl Acad Sci U S A 98, 12215-12220.

Pan, Y., Liu, G., Yang, H., Tian, Y. \& Tan, H. (2009). The pleiotropic regulator AdpA-L directly controls the pathway-specific activator of nikkomycin biosynthesis in Streptomyces ansochromogenes. Mol Microbiol 72, 710-723.

Pulsawat, N., Kitani, S. \& Nihira, T. (2007). Characterization of biosynthetic gene cluster for the production of virginiamycin M, a streptogramin type A antibiotic, in Streptomyces virginiae. Gene 393, 31-42.

Pulsawat, N., Kitani, S., Fukushima, E. \& Nihira, T. (2009). Hierarchical control of virginiamycin production in Streptomyces virginiae by three pathway-specific regulators: VmsS, VmsT and VmsR. Microbiology 155, 1250-1259.

Strohl, W. R. (1992). Compilation and analysis of DNA sequences associated with apparent streptomycete promoters. Nucleic Acids Res 20, 961-974.

Takano, E. (2006). $\gamma$-Butyrolactones: Streptomyces signalling molecules regulating antibiotic production and differentiation. Curr Opin Microbiol 9, 287-294.

Takano, E., Kinoshita, H., Mersinias, V., Bucca, G., Hotchkiss, G., Nihira, T., Smith, C. P., Bibb, M., Wohlleben, W. \& Chater, K. (2005). A bacterial hormone (the SCB1) directly controls the expression of a pathway-specific regulatory gene in the cryptic type I polyketide biosynthetic gene cluster of Streptomyces coelicolor. Mol Microbiol 56, 465-479.

Traag, B. A. \& van Wezel, G. P. (2008). The SsgA-like proteins in actinomycetes: small proteins up to a big task. Antonie van Leeuwenhoek 94, 85-97.

van Wezel, G. P., van der Meulen, J., Kawamoto, S., Luiten, R. G., Koerten, H. K. \& Kraal, B. (2000). ssgA is essential for sporulation of Streptomyces coelicolor A3(2) and affects hyphal development by stimulating septum formation. J Bacteriol 182, 5653-5662.

Wei, X., Yunxiang, L. \& Yinghua, Z. (2006). Enhancement and selective production of oligomycin through inactivation of avermectin's starter unit in Streptomyces avermitilis. Biotechnol Lett 28, 911-916.

Xu, H., Chater, K. F., Deng, Z. \& Tao, M. (2008). A cellulose synthase-like protein involved in hyphal tip growth and morphological differentiation in streptomyces. J Bacteriol 190, 4971-4978.

Edited by: J.-H. Roe 